\title{
Considerações relevantes para o ensino online durante a pandemia de Covid-19 nas escolas públicas do Amapá
}

\author{
Aldrin Santana \\ Artes Visuais, Universidade \\ Federal do Amapá Rod. Juscelino \\ Kubitschek, km 02 - Jardim Marco \\ Zero, Macapá/AP, 68903-419 \\ aldrin.santana@gmail.com
}

\author{
Jeovani Costa \\ Secretaria de Educação do Estado \\ do Amapá \\ Avenida FAB, 96 - Centro, \\ Macapá/AP, 68900-073 \\ jeovaniap13@gmail.com
}

\author{
Simey Castro \\ Secretaria de Educação do Estado \\ do Amapá \\ Avenida FAB, 96 - Centro, \\ Macapá/AP, 68900-073 \\ simeyguedes@hotmail.com
}

\begin{abstract}
This work has the theme: Relevant considerations for inline teaching during the COVID-19 pandemic in Public Schools in Amapá. It may be considered that the online teaching has been a challenge for the teacher and for the student, because the difficulties are innumerable: Internet access, cellphone or computer. The fact is that no one was prepared for this situation, nor the Amapá Secretariat of Education, as well as school managers, teachers students andtheir families. Everyone had to use creativity to develop students' learning process. Thus, this new modality requires reflection on the consideration of relevance that can contribute to education quality. These considerations highlight the importance and the family compromise at this moment to encourage the children to attend classes; teachers can create alternatives at websites, grous at social media and messengers' apps; schools to offer tools to teachers and students in this interaction and the State Secretariat of Education providing support to enable the necessary conditions to minimize the impact suffered in relation to the students' content and learning. Due to a certain part of the students not having access to the Internet, one of the alternatives found was delivering the activities on printed material. Our objective is knowing the difficulties presented during this period of pandemic in online education for the actors of the process and the expected solutions by the Educational System with the schools. The methodology used to carry out the work is based on bibliographic research. Therefore, it was found that innovation and creativity was means that the school was able to involve students so that they did not distance themselves, not necessarily using the technology, but making them part of the process and promoting reflection about their maturity in distance learning. From the above, it is concluded the importance of the Amapá StateSecretariat of Education, of the school, of the theacher and the family to develop the potential of
\end{abstract}

In: VII Workshop “O Futuro da Videocolaboração” (WCT-Video 2020), Online. Anais Estendidos do Simpósio Brasileiro de Sistemas Multimídia e Web (WebMedia). Porto Alegre: Sociedade Brasileira de Computação, 2020. () 2020 SBC - Sociedade Brasileira de Computação. ISSN: $2596-1683$ each student and to prepare them for the development of their knowledge and learning.

\section{KEYWORDS}

Online education, COVID - 19, Secretariat of Education of Amapá State

\section{Introdução}

Nos últimos meses, o Brasil e o mundo, têm enfrentado grandes dificuldades em virtude da crise sanitária causada pela COVID-19. Em 1918, outra pandemia trouxe grandes desafios para a humanidade, quando aconteceu a pandemia da gripe espanhola, que infectou mais de 500 milhões de pessoas [12]. Naquela época, também foi desafiador. O mundo não esperava que no ano de 2020 também teríamos que conviver com um vírus poderoso que em poucos meses devastou o mundo todo, fechou as portas das indústrias, comércios, lojas, lanchonetes e outros, para que fosse possível sobreviver. Com isso, buscou-se estratégias de reduzir a disseminação do vírus, as medidas de distanciamento social, além do incentivo à higienização das mãos e ao uso de máscara facial caseira, foram adotadas na maioria dos países, inclusive no Brasil. Embora não se saiba exatamente quando deixarão de ser necessárias, é importante que a população cumpra seu papel e as recomendações dos órgãos competentes. Sabe-se que a medida universal é o isolamento social, dada a sua importância para diminuir o avanço do vírus, como também a higiene pessoal para evitar tal propagação.

Não foi diferente na área da educação. Em meados de março, as instituições escolares foram afetadas e tiveram que suspender as aulas escolares presenciais, atingindo milhões de estudantes em todo o país. Com isso, no estado do Amapá, a secretaria de educação teve que acatar os decretos, tanto estadual (Decreto $\mathrm{n}^{\circ}$ $1.377 / 20$ ), quanto municipal (Decreto $\mathrm{n}^{\circ} 1.656 / 2020$ ) e interromper as aulas em todas as escolas. Apesar das circunstâncias, as escolas juntamente com a secretaria de educação estão em uma força conjunta para que os alunos não sejam tão prejudicados no ensino e na aprendizagem. A suspensão das aulas foi uma medida necessária para se evitar a propagação da contaminação, tendo em 
vista que a escola é um ambiente facilitador de contaminação devido a aglomeração de pessoas, de acordo com a Organização Mundial de Saúde - OMS. Dessa maneira, o Brasil tem acompanhado o formato mundial e as escolas passaram a desempenhar com os alunos as atividades pedagógicas no formato à distância.

Atualmente, a Secretaria de Educação do Estado do Amapá, junto com as escolas estaduais, com muitas dificuldades, conseguiu avançar no sentido de disponibilizar meios como: plataformas online, aulas ao vivo em redes sociais e envio de materiais digitais aos alunos.

"As aulas on-line são disponibilizadas por meio da Escola Virtual do SIGEDUC, Plataformas Educacionais disponíveis no Portal Aprendizagem em Casa, redes sociais, WhatsApp, aulas gravadas, entre outras ferramentas tecnológicas; e na ausência da Internet, apostilas estão sendo disponibilizadas e utilização do livro didático”. [8]

Diante da situação, o ensino remoto virou o novo "normal". As escolas do Amapá tiveram que se adaptar ao novo modelo para oferecer aos alunos práticas de aprendizagem de forma remota e condições de estudos. Com isso, a Secretaria de Estado de Educação, por meio dos gestores e professores, buscou formas e alternativas de minimizar as perdas pelos quais todos estão passando.

Os professores das escolas públicas estaduais tiveram que se reinventar, transformar suas metodologias de ensino, tornar as aulas interativas, incluir novos conteúdos, utilizar novas ferramentas de ensino, adaptar as aulas práticas ao formato online, incluir recursos visuais e readequar as atividades e os materiais didáticos.

\section{Considerações relevantes para o ensino online durante a pandemia}

É importante considerar os dados relevantes frente ao cenário que população educacional está enfrentando, no qual a Secretaria Estadual de Educação teve que tomar decisões precisas como: disponibilizar por meio da Escola Virtual do SIGEDUC, Plataformas Educacionais disponíveis no Portal Aprendizagem em Casa, redes sociais, WhatsApp, aulas gravadas, entre outras ferramentas tecnológicas, para dirimir as questões complexas na área educacional frente a esse cenário que o Amapá está passando junto os outros estados do Brasil.

A presente tentativa em buscar condições, propiciar caminhos e estratégias possíveis de serem realizadas para que o aluno consiga ter a oportunidade de aprender está sendo de grande importância para diminuir o impacto causado nesse período em que o aluno não está frequentando as aulas presencias, mas está, de uma forma ou de outra, recebendo o ensino, mesmo que deficitário em função das inúmeras dificuldades apresentadas no decorrer dessa pandemia. Diante disso, é que o Sistema Educacional de Ensino busca impedir a evasão dos alunos, em razão da atual situação em que as escolas estão enfrentando. E com todas as dificuldades, que tanto os professores, quanto os alunos sentem para que as aulas possam ser realizadas, percebe-se que existe uma grande força de vontade em não desistir tanto em ensinar, como em aprender.

A Secretaria de Estado da Educação do Amapá está amparada pela resolução do Conselho Estadual de Educação de nº 033/2020, no art. 1.

\begin{abstract}
"Estabelecer o regime especial de atividades escolares não presenciais, para fins de cumprimento do calendário letivo do ano de 2020 em consonância com a Medida Provisória 934/2020, no que tange ao cumprimento da carga horária mínima estabelecida de oitocentas horas, definido essencialmente pela manutenção das atividades pedagógicas sem a presença de estudantes e professores nas dependências escolares, no âmbito de todas as instituições ou redes de ensino públicas e privadas, da Educação Básica, Profissional e Superior, pertencentes ao Sistema Estadual de Educação do Amapá”. [5]
\end{abstract}

E também, através do Núcleo de Inspeção e Organização Escolar-NIOE/SEED que realizou orientações para a elaboração do plano de ação utilizando a metodologia de atividades pedagógicas não presenciais (aulas programadas), e considerando a atual situação, solicitou aos professores que fazem parte do quadro estadual de educação do Amapá e os professores federais a disposição do estado do Amapá que realizassem a elaboração de um plano de ação exequível, estabelecendo alternativas que possam nortear e auxiliar na execução da ação educativa para garantir o ensino/aprendizagem aos estudantes atendendo a realidade das escolas do Sistema Estadual de Ensino do Estado do Amapá.

É importante salientar que dentre essas orientações pedagógicas não presencial, foi observado a recomendação do uso ou não das tecnologias como: vídeo/aula (esse não sendo obrigatório para o professor), a secretaria de educação informa em eu site que colocou à disposição dos estabelecimentos de ensino a Plataforma Escola Virtual-EVirtual, onde disponibiliza os conteúdos, canal de TV, vídeo, material impresso e o livro didático e ainda teve a preocupação de normatizar por meio de um guia de orientação para implementar as atividades pedagógicas não presenciais para que os gestores, pedagogos, professores e pais assegurassem os direitos de aprendizagens dos alunos ao longo desse período pandêmico nas etapas e modalidades da Educação Básica. Assim, em consonância com a Resolução № 033/2020 $\mathrm{CEE} / \mathrm{AP}$, que estabelece orientações para direcionar as instituições de ensino na forma de computar o mínimo de carga horária de oitocentas horas estabelecidas em conformidade com a Medida Provisória no 934/20 - Governo Federal.

A Secretaria de Estado da Educação do Amapá, sentindo a necessidade de normatizar as ações relacionada as atividades escolares, resolveu instituir a portaria 036/2020 que informa no art. $3^{\circ}$ que "Fica determinado a suspensão de todas as atividades administrativas e pedagógicas nas unidades escolares, no período de 15 dias, conforme o estabelecido no decreto $n^{\circ} 1414 / 2020-$ GEA" [13], conforme o último decreto $\mathrm{n}^{\circ} 2.908$, de 31 de agosto de 2020 [9], instituído pelo governador do Estado do Amapá que 
Considerações relevantes para o ensino online durante a pandemia do covid-19 nas escolas públicas do amapá

prorrogou por mais 30 dias as medidas de distanciamento adotadas diante da pandemia.

Atualmente, com base nos relatórios epidemiológico e parecer científico da Vigilância em Saúde e Sanitária, o governo do estado não retorna as atividades presenciais por uma garantia da não permissão de potencializar um possível aumento da contaminação do corona vírus no estado.

Nesse sentido, este artigo poderá servir como discussão sobre os desafios e as dificuldades apresentadas nesse período pandêmico no ensino online para os atores do processo e as soluções apresentadas pelo Sistema Educacional junto as escolas.

\section{Considerações relevantes para o ensino pós pandemia}

No retorno das aulas presenciais, teremos a possibilidade de entender o real impacto da COVID-19 na educação. Além do impacto causado pela perda familiar, como também o sofrimento que grande parte da população sentiu em relação a perda da renda familiar, gerando vários sentimentos tanto para o aluno, como para o professor. Após longo período de isolamento, grandes lições serão apreendidas em relação à educação online, no qual será possível perceber que se pode ir além dos muros da escola e ter a oportunidade de inovar a metodologia de ensino com diversos recursos atrativos para o aluno, como também o papel fundamental da família na educação dos filhos.

Neste contexto, vale ressaltar a importância da formação continuada e o aperfeiçoamento profissional do educador para o uso pedagógico das tecnologias educacionais, de forma a auxiliar na prática de suas aulas, haja visto que, nesse período de aulas remotas, os profissionais da educação tiveram papel fundamental no processo ensino aprendizagem do aluno, no qual apresentaram engajamento e esforço na aplicabilidade das aulas.

Diante disso, é necessário no processo de retorno às atividades presenciais, que a escola esteja preparada, com condições de receber a clientela escolar, obedecendo as medidas de segurança sanitárias propostas pela Organização Mundial da Saúde e Vigilância Sanitária, oferecendo a aferição de temperatura aos profissionais da educação e alunos, disponibilizando álcool em gel nos ambientes da escola, dividindo e reorganizando as turmas de forma escalonada com a garantia de distanciamento social mínimo, dentro das salas de aula, cobrando o uso obrigatório das máscaras e mantendo a higienização de todos os ambientes.

Para regulamentar este retorno as aulas presenciais, o Conselho Estadual de Educação, por meio da nota técnica 01/2020$\mathrm{CEE} / \mathrm{AP}$, normatizou para subsidiar as decisões pelas redes e instituições de ensino do Estado do Amapá, acerca da reorganização dos calendários para a possível reabertura das escolas.

Sendo assim, essa organização estrutural da educação deverá atingir objetivos de aprendizagem qualitativa, visando não prejudicar os alunos, porém mesmo diante do cenário de pandemia, deverão prosseguir seus estudos sem perdas de carga horária mínima prevista, pois muitos dependem desse
Anais Estendidos do WebMedia'2020, Online, Brasil

cumprimento para efetivação e participação no ENEM. Dessa forma, o Conselho Nacional de Educação, se manifestou através do Parecer $n^{\circ}$ 5/2020.

\begin{abstract}
"concerne a se assegurar que a reposição de aulas e a realização de atividades escolares possam ser efetivadas de forma que se preserve o padrão de qualidade previsto no inciso IX do artigo $3^{\circ}$ da LDB e artigo 206 da $\mathrm{CF} / 88$ garantindo o atendimento aos princípios de condições e permanência na escola; liberdade de aprender e ensinar, pesquisar e divulgar o pensamento, a arte e o saber; pluralismo de ideias e concepções pedagógicas, bem como a contínua valorização dos profissionais da educação, a gestão democrática do ensino público, dentre outros”. [7]
\end{abstract}

\section{Visão de futuro na área de videocolaboração na educação online}

O tempo de pandemia pelo Corona vírus COVID 19, com o isolamento social, fez com que as pessoas refletissem e buscassem uma nova ressignificação para suas vidas, em meio as dificuldades apresentadas, a educação presencial deixou de existir, a comunicação digital ganhou força, e com isso a educação remota teve seu papel em destaque, vale ressaltar que uma nova modalidade está sendo adotada pelas instituições de ensino como meio de proporcionar mecanismos que atendam às necessidades dos educandos e professores, a exemplo o ensino híbrido, que é uma metodologia que está associada ao aprendizado online com o off-line, momento em que o educando estuda sozinho, de maneira virtual, e também em outro dado momento estuda interagindo com outros educandos.

Observa-se que as tecnologias do futuro tendem na direção do vídeo colaboração, da comunicação interativa e audiovisual. Neste contexto, a tendência é o uso de várias ferramentas que além de poder ser usada para a transmissão de aulas, tem recursos que possibilitam a reunião dos estudantes em salas virtuais específicas, o envio e recebimento de atividades, calendário, chat, além de diversos recursos de integração com diversos outros aplicativos.

Pode-se observar que as tecnologias do futuro próximo vêm trazer a interatividade de forma instantânea, na educação não será diferente, a pós pandemia trará um outro olhar com relação a educação online, os alunos aprenderão com orientação de seus professores, e também por meio da tecnologia digital, integrando a televisão, a Internet e a telefonia digital, novas mídias cada vez mais presente e sua evolução terá um caráter irreversível.

A partir de agora, os professores necessitam repensar o processo, os gestores por sua vez devem permitir o êxito desse processo e os alunos precisam se ajustar ao novo modelo de aprendizagem. Existe uma imensidade de recursos tecnológicos disponíveis, que facilitam a relação e a troca de saberes nunca antes imaginada.

Portanto, não há como pensar em uma educação absolutamente presencial e desconsiderar todas as possibilidades que o digital pode acrescentar. O Brasil e o mundo constataram que o futuro já chegou exigindo inovação e mudança, iniciando uma nova era, pós pandemia, com a educação combinada aos 
novos desafios, preparando nossos educandos para uma vida inovadora, desafiante, conectada e compartilhada.

\section{Considerações finais}

O referido artigo buscou um olhar diferenciado em relação aos esforços da Secretaria de Estado da Educação, dos profissionais da educação, dos educandos e da família frente ao ensino remoto. As dificuldades foram sentidas por todos os atores, principalmente pelos professores que tiveram que se reinventar, além de lidar com pressão de adaptar-se a ferramentas virtuais, de preparar atividades para manter os alunos estimulados e, ao mesmo tempo, estar disponíveis para esclarecer dúvidas.

Mais do que isso, a Secretaria de Educação do Estado do Amapá teve a preocupação em oferecer aos professores por meio da Escola Virtual do SIGEDUC, a Plataforma Escola Virtual EVirtual, como ferramenta para realização das atividades de ensino e de interação/comunicação entre gestores, coordenadores pedagógicos, professores e estudantes, além de fazer o acompanhamento das aulas, por meio dos relatórios semanais de desempenho dos alunos.

Como em todo momento novo, é necessário estar aberto à mudança. Por isso, todos devemos apresentar prudência em tudo que é feito, pois estávamos acostumados com uma rotina pedagógica estabilizada e agora além dos conteúdos que ensinamos, temos que extrapolar os conceitos contidos nos livros buscando aprender juntos e superar os desafios.

Ao longo desse período, as estratégias do ensino a distância cumprem papel importante para a redução dos efeitos negativos do distanciamento temporário, mas as evidências indicam que lacunas de diversas naturezas serão criadas. Com isso, o Conselho Estadual de Educação tem papel fundamental para realizar as normatizações para cumprimento do ano letivo, desde já, as redes de ensino precisam começar a planejar um conjunto robusto de ações para o retorno das aulas, adotando medidas de ordem sanitária, visando a saúde e o bem-estar de todos que fazem parte do processo educacional.

Enfim, a lição que se tira é que não necessitamos ter somente salas de aula, como se fosse a única maneira para disponibilizar os conteúdos para os alunos, mas, é possível trabalhar com qualidade, usando as plataformas virtuais, além de promover a formação continuada e o aperfeiçoamento profissional do uso das tecnologias educacionais aos professores. O sistema educacional precisa preparar as pessoas para o século XXI. Dessa forma, transformar a educação de fato de qualidade.

\section{REFERÊNCIAS}

[1] Agência Brasil, 2020, Escolas estaduais começam estudos online no Rio de Janeiro, https://agenciabrasil.ebc.com.br/educacao/noticia/202003/escolasestaduais-comecam-estudos-online-no-rio-de-janeiro.

[2] Banco Mundial (2020). Políticas educacionais na pandemia da COVID-19: o que o Brasil pode aprender com o resto do mundo?

[3] C. Campos (2020). Educação e Covid-19: Um levantamento das respostas de órgãos federais e estaduais à epidemia.

[4] CEE, 2020, Nota Técnica ${ }^{\circ}$ 01/2020, https://cee.portal.ap.gov.br.

[5] CEE, 2020, Resolução n ${ }^{\circ}$ 033/2020, https://cee.portal.ap.gov.br.

[6] C. Costin et al (2020). A escola na pandemia: 9 visões sobre a crise do ensino durante o coronavirus. Porto Alegre: Ed. do Autor.

[7] Conselho Nacional de Educação (CNE), 2020, Reorganização do Calendário Escolar e da possibilidade de cômputo de atividades não presenciais para fins de cumprimento da carga horária mínima anual, em razão da Pandemia da COVID-19, PARECER CNE No: 5/2020.

[8] G1 AP, 2020, Educação na pandemia: $20 \%$ dos estudantes da rede estadual do $\mathrm{AP}$ não têm acesso às aulas remotas, https://g1.globo.com/ap/amapa/noticia/ 2020/07/06/educacao-na-pandemia-20percent-dos-estudantes-da-redeestadual-do-ap-nao-tem-acesso-as-aulas-remotas.ghtml.

[9] GEA, 2020, Decreto ${ }^{\circ} 2.908$, https://www.legisweb.com.br/legislacao/?id=400740\#: :text=Ficam\%20suspens as\%20as\%20aulas\%20presenciais,01\%20de $\% 20$ setembro\%20de $\% 202020$.

[10] MEC, 2020, Conselho Nacional de Educação esclarece principais dúvidas sobre o ensino no País durante pandemia do coronavírus, http://portal.mec.gov.br/ busca-geral/12-noticias/acoes-programas-e-projetos-637152388/87161conselho-nacional-de-educacao-esclarece-principais-duvidas-sobre-o-ensinono-pais-durante-pandemia-do-coronavirus.

[11] MEC, 2020, O que é educação a distância? http://portal.mec.gov.br/escola-degestores-da-educacao-basica/355-perguntas-frequentes-911936531/educacao-adistancia-1651636927/12823-o-que-e-educacao-a-distancia.

[12] J. K. Taubenberger and D.M. Morens, 2006, 1918 Influenza: the mother of all pandemics. Emerging Infectious Diseases, https://www.ncbi.nlm.nih.gov/pmc/articles/PMC3291398.

[13] SEED, 2020, Portaria No 036/2020 - SEED, https://editor.amapa.gov.br/arquivos_portais/publicacoes/EDUCA\%C3\%A7\%C3 \%A3O_badc965aa1733ba2b82fe2e18a7e9fc0.pdf

[14] Todos pela educação (2020). Nota Técnica. Ensino a distância na educação básica frente à pandemia da covid- 19 . 